\title{
Interactions of Coloured Heavy Stable Particles in Matter
}

\author{
Rasmus Mackeprang \\ (Niels Bohr Institute, Denmark) \\ Andrea Rizzi \\ (INFN Pisa,Italy)
}

September 25, 2018

\begin{abstract}
In this paper we present a physics model for the interactions of stable heavy hadrons containing a heavy parton with matter. The model presented is a natural continuation of the work started in [1. However, changes and generalisations have been made allowing for the description of a broader scope of physics scenarios. As a special case the model is tested on the cases of stable gluino and stop hadrons, thus covering both stable colour triplet and octet states. Conclusions are drawn regarding the phenomenology of these cases including how to distinguish between them.
\end{abstract}

\section{Introduction}

Many extensions to the Standard Model (SM) feature long-lived particles carrying SM charges. Most prominently Supersymmetry (SUSY) models exist where the lightest supersymmetric particle (LSP) carries either electromagnetic or colour charge. Also more generic models incorporating hidden valleys and global symmetries exist which allow for similar phenomenological consequences ([2], [3]). The particle contents and possible interactions of different physics scenarios leads to differences in the energy deposition characteristics of the heavy hadronic states. In this paper we demonstrate that these differences can be used as a powerful tool to discriminate between models.

Section 2 contains a brief summary of contemporary models providing long-lived or even stable strongly interacting particles. The models listed in no way comprise a complete list.

The interactions of stable, heavy and coloured particles differ dramatically to those of the SM particles. For the future possible discovery of such particles it is therefore crucial to have an understanding of their interactions with matter. In section 3 we present a toolkit for simulation of the interaction of heavy quasi-stable coloured particles with matter. The physics treatment follows that of the gluino R-hadron study presented in [1] with the difference being the generalisation to a broader physics scope 
and the implementation into Geant4 4. Also the kinematical treatment of secondaries from nuclear interactions differs between the two models. The structure of the Geant 4 framework enables us to easily study heavy colour triplet and octet states.

Simple phenomenological conclusions for both types of states are derived from our simulations and presented in section 4 while the reader is referred to [5] for usage documentation. The main difference between colour triplet and octet states turns out to be the relation between the electromagnetic and the hadronic energy loss and the shape of the resulting $\mathrm{dE} / \mathrm{dx}$-distributions as well as their absolute normalisations. In the case of models where the heavy states are produced in pairs more information may of course be deduced from event variables such as charge correlations between the two particles.

\section{Models with heavy stable coloured par- ticles}

Several extensions to the SM predict new stable or quasi-stable interacting particles with high masses. From a collider experiment point of view quasi-stable particles, i.e. particles decaying with a $c \tau$ at the order of a few metres, can be regarded as stable. It is straightforward to include the interaction with matter of heavy stable particles that only have electromagnetic interactions (like stable sleptons) in a detector simulation. The interaction of coloured particles, however, is not modelled in the current version of the main high energy physics simulation toolkit, Geant4.

In the following we will provide some examples of theoretical models predicting heavy stable (or at least long-lived) coloured particles. A comprehensive overview of different models may be found in [6]. Whereas the interaction model presented in section 3 does not depend on the specific physics scenario, this may indeed affect the production cross section and kinematic distributions.

\subsection{Split Supersymmetry}

Long-lived gluinos are predicted by several supersymmetric models [7] in which the gluino is the Lightest Supersymmetric Particle (LSP) or the Next to LSP (NLSP). It is therefore by R-parity stable or quasi-stable. Recently the Split supersymmetry model [8] has predicted potentially long-lived gluinos with a mass of order $1 \mathrm{TeV} / \mathrm{c}^{2}$. In this scenario the decay of the gluino into the LSP together with a quark anti-quark pair is heavily suppressed by a very high squark mass. Gluinos in this mass range may be produced in the near future at the LHC proton-proton collider and, being coloured, they would hadronise into heavy charged or neutral bound states. Such hadrons are commonly referred to as R-hadrons as their stability is rooted in R-parity. R-hadrons can be of several types: gluon-gluino balls, gluino R-baryon and gluino R-meson. The interaction of gluino R-hadrons with matter has already been studied in [1] and implemented in a Geant3 toolkit, so we will focus on R-hadrons in the 
Figure 1: The decay of a gluino in Split SUSY. The mass term in the squark propagator suppresses the decay.

demonstration of the implemented Geant4 model to easily compare the results. Some Monte Carlo generators already exist [9, [10, that are able to simulate the production and subsequent hadronisation of gluinos. These programs in connection with the toolkit presented here make it possible to study the full range of observables of R-hadron events as they might appear in a detector.

\subsection{Other models}

Another model in which a heavy coloured particle is stable is the extra dimensional SUSY scenario proposed in 11. In this model the supersymmetry breaking is obtained with a bound condition on a compactified extra dimension and the predicted LSP is, in a large fraction of the parameter space, the stop. As is the case for gluinos the stop may be produced at LHC and will hadronise in baryon and meson like states in which one quark or anti-quark is replaced with the stop or anti stop. Also non supersymmetric models exists in which stable massive coloured particles are expected. Universal Extra Dimension (UED) scenarios may contain stable or quasi-stable Kaluza Klein excitations [12, [13. Specifically if the KK number is not violated (or weakly violated) the excitations may be produced in pairs and they can then be stable or long lived.

\section{$3 \quad$ Interaction Model in Geant4}

\subsection{Geant4 framework}

Geant4 is an Object Oriented physics simulation toolkit that is the current standard for HEP experiments. The previous version, Geant3, was FORTRAN based and was extensively used by LEP experiments and in the preparation of the LHC experiments. Due to Geant4 being an OO toolkit the interaction model was implemented as a simple add-on. This meant that no modifications to the core of the toolkit were required. The implementation included adding new particles (e.g. R-hadrons) and their corresponding interaction processes. A particle in Geant4 is represented by a pair of objects, a G4ParticleDefinition and a G4DynamicParticle. The G4ParticleDefinition contains the static information about the parti- 
cle type such as name, mass, PDG code, spin etc. The G4DynamicParticle contains the four momentum of a specific particle in an event. The new package adds particle definitions for all particles in the relevant physics scenario. The particles are defined from a file that contains PDG-code of the particle, mass and name in a Susy Les Houches Accord (SLHA) like format [14. The particle definitions are all instances of a class called CustomParticle that not only contains the normal static information but also allows the splitting of the particle into a heavy parton (e.g. a gluino) and a light quark system (LQS). This is used to facilitate the treatment of interactions with matter in an easy and correct fashion. The interactions with matter are described in a so called G4Process. A dedicated G4Process has been implemented as described in following sections. In order to let Geant4 know of this process it should be registered to the Geant 4 framework for each particle that undergoes this process. This must be done when compiling, but a great amount of flexibility may be retained for subsequent run-time reconfiguration.

\subsection{Cross Section}

Barring one extension, the cross section calculation follows closely that of 1]. We therefore refer to that paper for a thorough review.

The basic idea is to use a purely geometric cross section tuned to $\pi-p$ scattering. The heavy parton is neglected in this context. As the spatial extent of a wave function scales as $1 / M^{2}$, the cross section contribution becomes negligible. In this picture we are thus considering the heavy parton to be exclusively a reservoir of kinetic energy. In contrast, each light valence quark is assigned a total cross section of 12 mbarn per nucleon leading, for instance, to a value of 24 mbarn for a meson carrying a colour octet heavy parton (e.g. a gluino R-meson). This value stems from the fact that the size of the total cross section should be matched to the high energy limit of the total $\pi-p$ cross section. The contribution for an $s$ quark is set to 6 mbarn to conform with 11. For the translation of a cross section per nucleon, $\sigma_{n / p}$, into a cross section per nucleus, $\sigma_{A}$, the same GHEISHA convention [15] is used as in the Geant3 work. The relation between the two cross sections is:

$$
\sigma_{A}=1.25 \times \sigma_{n / p} \times A^{0.7}
$$

One additional feature that has been introduced is the ability to add to the flat cross section a non-relativistic Breit-Wiegner resonance. The user may at run-time specify resonance position, width and height as shown in figure 2

\subsection{Choice of Final State}

The choice of final state is made using a slightly modified decision tree with respect to the one used in the Geant3 work, although the end result is the same. The decision tree is an iteration over the following steps until a process is selected.

- Populate a list of possible processes (positive Q-value) [5] 


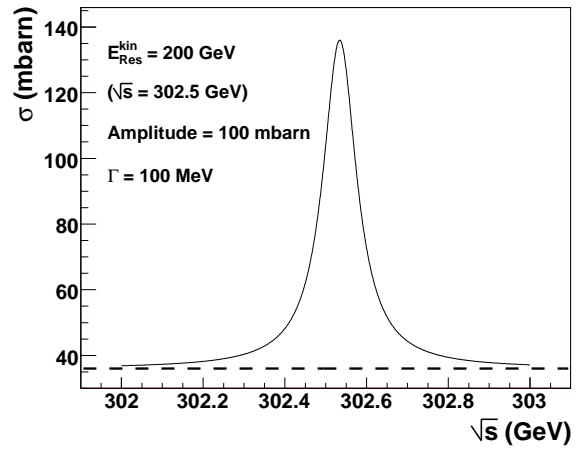

(a) In Centre of Mass System (CMS)

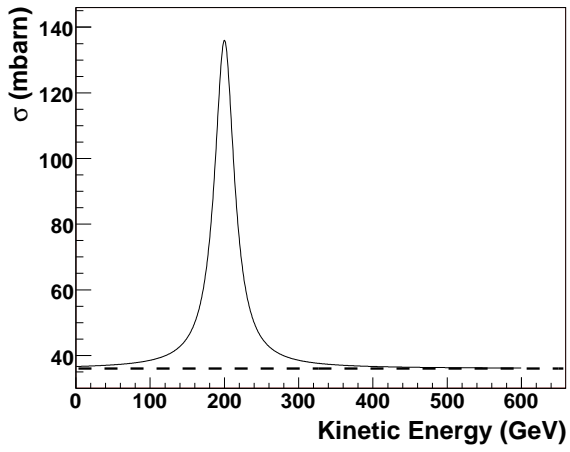

(b) In labframe

Figure 2: An example showing how a resonance could be configured. This example is a gluino R-baryon $\left(m_{\tilde{g}}=300 \mathrm{GeV} / \mathrm{c}^{2}\right)$ with a base cross section of 36 mbarn and a $100 \mathrm{mbarn}$ resonance added.

- Assign $2 \rightarrow 2$ and $2 \rightarrow 3$ processes a priori relative probabilities of $15 \%$ and $85 \%$ respectively, as elastic scattering amounts to approximately $15 \%$ of the total cross section for light hadrons at high momenta.

- Choose a process at random, given the a priori probabilities

- For $2 \rightarrow 3$ processes, a phase space function decides the probability of the process being accepted.

The phase space function describes the phase space fraction available for $2 \rightarrow 3$ scattering. It is defined using the same expression as in Geant3:

$$
F(Q)=\frac{\sqrt{1+\frac{Q}{2 m_{\pi}}}\left(\frac{Q}{Q_{0}}\right)^{3 / 2}}{1+\sqrt{1+\frac{Q}{2 m_{\pi}}}\left(\frac{Q}{Q_{0}}\right)^{3 / 2}}
$$

This choice of functional expression is motivated by the fact that the function is, by Lorentz invariance, expected to be a function of $Q=$ $\sqrt{s}-\sum_{i} m_{i}$ where $i$ enumerates the final state particles. As an ansatz one may use the expression

$$
F(Q)=\frac{\frac{\mathrm{d} \phi_{3}(Q)}{\mathrm{d} \phi_{3}\left(Q_{0}\right)}}{\frac{\mathrm{d} \phi_{2}(Q)}{\mathrm{d} \phi_{2}\left(Q_{0}\right)}+\frac{\mathrm{d} \phi_{3}(Q)}{\mathrm{d} \phi_{3}\left(Q_{0}\right)}}
$$

where $\mathrm{d} \phi_{N}(Q)$ denotes the absolute phase space for N-body scattering at a scale $Q$. Note that the phase spaces are normalised to a scale $Q_{0}$ due to the fact that they have different dimension. The actual derivation of equation (2) is rather involved [16] and involves some approximations. The value of $Q_{0}$ is determined by imposing that $F\left(Q_{0}\right)=0.5$ as required in equation (3). It turns out that the value becomes $1.1 \mathrm{GeV}$. F is shown as a function of some commonly used parameters in figure 3

The relative frequencies with which $2 \rightarrow 2$ and $2 \rightarrow 3$ processes are chosen may be seen in figure 4 

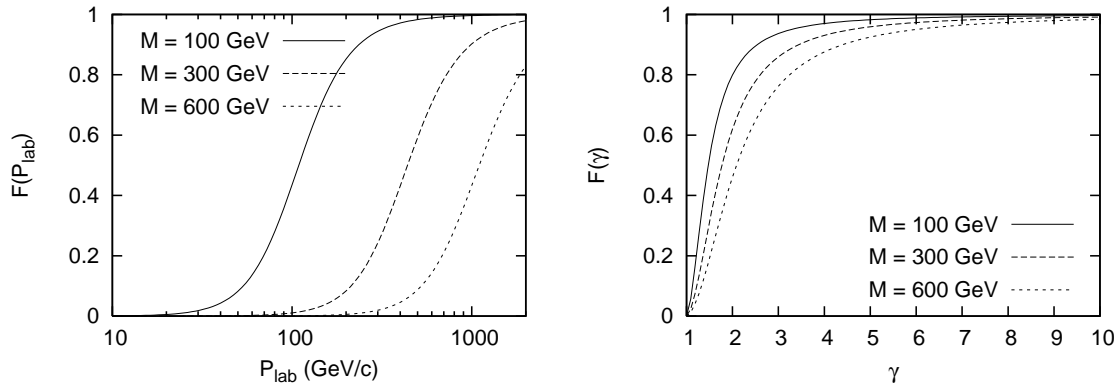

Figure 3: Phase space function $F(Q)$ shown in a few parametrisation.

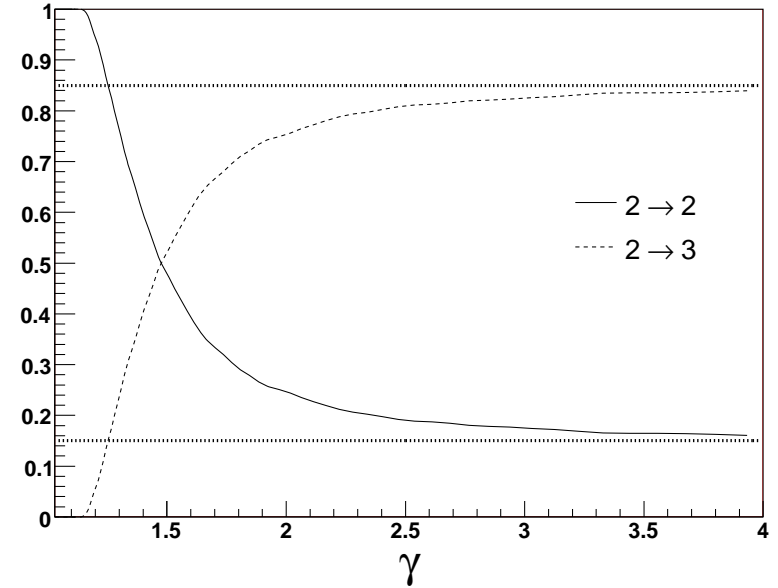

Figure 4: Fraction of $2 \rightarrow 2$ and $2 \rightarrow 3$ processes selected as a function of $\gamma$ of the incident particle. There is no discernible difference between different particle masses or types. Although this specific plot was made for $300 \mathrm{GeV} / \mathrm{c}^{2}$ gluino baryons in iron, stop hadrons at other masses in other materials yield the same result. 


\subsubsection{Charge Exchange Suppression}

In a nuclear interaction a hadron containing a heavy parton may interact through pomeron exchange or reggeon exchange. Pomeron exchange will leave the charge unaltered whereas Reggeon exchanges will allow the charge to be transferred.

In the above discussion no preference has been selected between reggeon and pomeron exchange. This is of course due to the fact that we have no a priori reason to assume a difference in strength between the two mechanisms. Theoretical arguments might appear, however, as to why one exchange mechanism should dominate over the other and so an additional feature has been added to supply a charge exchange suppression mechanism.

The mechanism is simply expressed as a probability of rejecting a process and rerun the final state selection if the selected final state changes the charge of the heavy hadron. Other ways to do this could be devised if theoretical basis could be found for a specific mechanism.

\subsection{Kinematics}

Once the final state of an interaction is selected, several things may occur. Decisions need to be taken as to how momenta are assigned to the outgoing particles. Also the struck nucleus may undergo some changes. These decisions are highly model dependent and two models are presented here.

The basic tenet of both models is that the interaction between the heavy hadron and the nucleon takes place via the LQS alone. This is a logical extension of the geometric cross section calculation in section 3.2 As the heavy parton in this formulation is strictly a reservoir of kinetic energy, it has been assumed that it will not interact. Rather the LQS is assumed to interact exclusively. Imposing the requirement that the LQS and the heavy parton are co-moving leads to the condition that the LQS carries the kinetic energy $E_{k i n, L Q S}=\frac{M_{L Q S}}{M_{\text {tot }}}$, leading to the treatment as a low-energy collision in spite of the high energy carried by the hadron as a whole.

This treatment is naturally only valid as long as $M_{L Q S} \ll M_{T o t}$.

\subsubsection{Simple Toy Model}

This model embodied in the "ToyModelHadronicProcess" is included in the toolkit for scenarios where the user wishes to make initial conclusions while minimising the "black box" of the machinery and thus maintaining an overview. It is hardly valid for physics conclusions but it is a useful tool to test the process lists supplied as it is the simplest ansatz possible for a kinematics treatment.

The collision is treated as that between a free nucleon and the LQS of the heavy hadron. Quasi-elastic $(2 \rightarrow 2)$ processes are handled by the boosting of the LQS and the target nucleon to the CMS and assigning the outgoing particles back to back momenta along a random axis. Momenta are then boosted back to the lab-frame. Upon creation of secondaries and assignment of momenta, the heavy parton and the LQS are re-coupled 
whilst imposing three momentum conservation and calculating the energy by keeping the heavy hadron on-shell. This re-coupling procedure results in an excess energy. The difference may be quantified by:

$$
\Delta E=E_{L Q S}+E_{H P}-\sqrt{\left(\vec{p}_{L Q S}+\vec{p}_{L Q S}\right)^{2}+M_{T o t}^{2}}
$$

This excess energy is added as a local energy deposit in Geant4. $2 \rightarrow 3$ processes are treated by generating the momenta as a flat distribution in a Dalitz plot. The same recombination procedure applies as for the $2 \rightarrow 2$ processes.

\subsubsection{Parametrised Model}

This model - implemented in the FullModelHadronicProcess - is based upon the parametrised model for light hadrons 17. with certain revisions made to the G4ReactionDynamics class to eliminate flavour dependence 18. As in the toy model, the heavy parton and the LQS are decoupled from one another, sharing the available kinetic energy. The quark system is then passed on to the parametrised model to generate the secondaries as well as the "black track particles". These black track particles are nuclear fragments from the struck nucleus such as deuterons, tritons or $\alpha$ particles created with very low energies.

As for the toy-model, the excess energy from the recombination is added as a local energy deposit by Geant4. This local energy deposit is shown in figure 5 for gluino hadrons as well as stop hadrons in iron and carbon.

It should be stressed that this is the only point at which the physics content of the model deviates from Geant3. In Geant3 the heavy hadron was treated as one heavy object in the kinematics treatment, and the kinematic distributions were rescaled to take into account that not all of the kinetic energy was available for production of secondaries. In the present work the reaction is viewed as being between a totally decoupled LQS and a nucleus with a subsequent re-coupling of the LQS with the heavy parton. All differences between Geant3 and Geant4 should thus be expected to have their origin in this difference of kinematical treatment.

\section{Results}

Process lists corresponding to the cases of stable gluino hadrons and stable stop hadrons were implemented assuming no oscillation in the stop case. No resonance or charge exchange suppression was configured.

The first observation that can be made from simply considering the possible processes is that a gluino meson or a stop mesonino undergoing nuclear reactions in matter may at some point acquire a baryon number, thus turning into a gluino baryon or a stop sbaryon respectively. In ordinary matter it is impossible for the heavy hadron to part with the acquired baryon number due to baryon number conservation. Conversely anti-stop sbaryons will tend to annihilate with nuclear matter leading to a conversion into mesoninos. Where not explicitly noted differently otherwise, the particles studied were gluino baryons and stop baryons. In the 


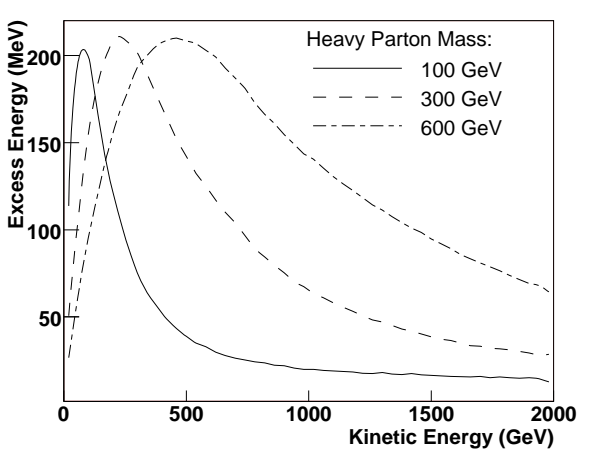

(a) Gluino hadrons in iron

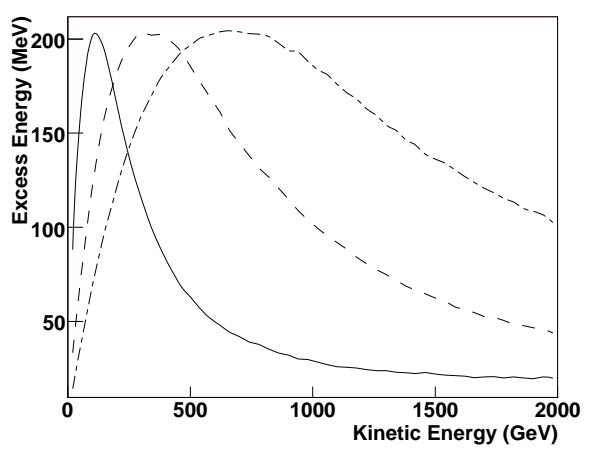

(c) Stop hadrons in iron

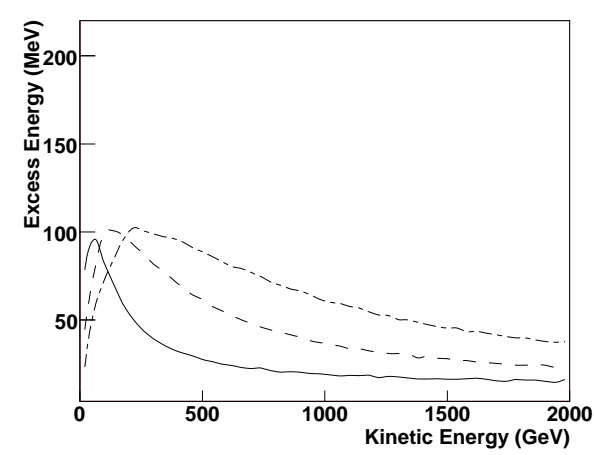

(b) Gluino hadrons in carbon

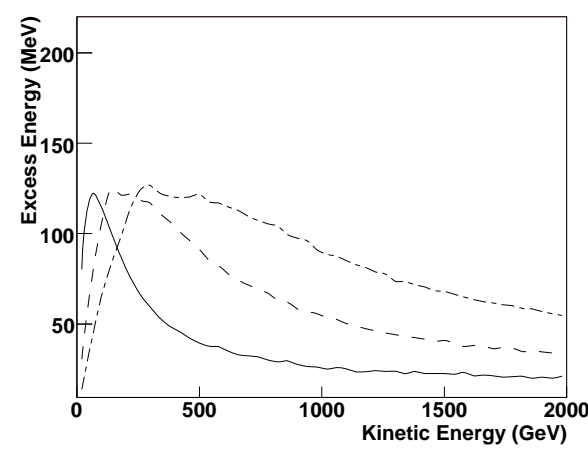

(d) Stop hadrons in carbon

Figure 5: Local energy deposit from momentum recombination. 
cases where anti-stops were explicitly studied the incident particle was chosen to be a anti-stop mesonino.

The focus in this section will be twofold. The compatibility of the results obtained with Geant4 to the Geant3 results will be demonstrated, and differences between the colour octet / colour singlet heavy parton cases will be highlighted.

\subsection{Baryonisation}

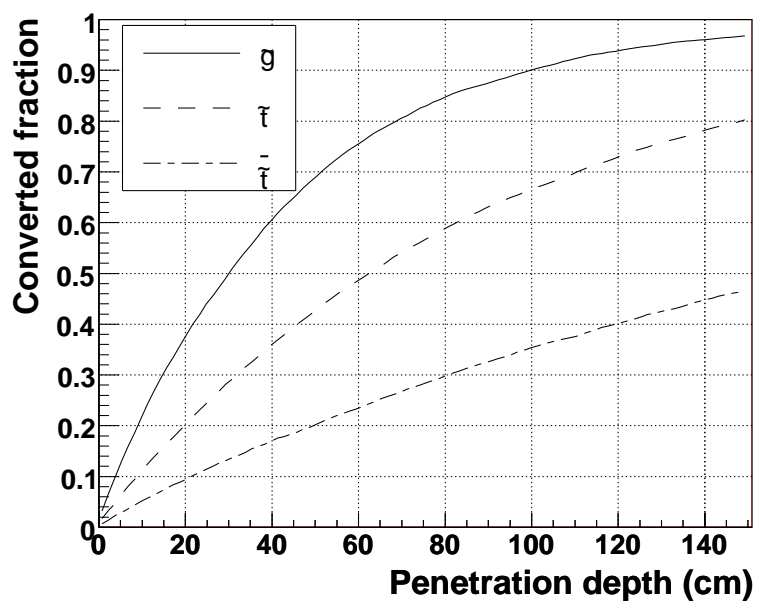

Figure 6: Fraction of gluino baryons, stop sbaryons and anti-stop mesoninos as a function of the travelled distance in Iron.

Figure 6 shows the fraction of gluino mesons, stop mesoninos and antistop sbaryons that have converted into gluino baryons, stop sbaryons or anti-stop menoninos respectively as a function of the travelled distance in iron. The heavy parton has been given a mass of $300 \mathrm{GeV} / \mathrm{c}^{2}$ but this has little influence as long as $M_{L Q S} \ll M_{T o t}$ due to the nature of the cross section calculation. There is no discernible difference between the toy-model and the parametrised model, as this quantity exclusively relies on pure phase space considerations. Any difference would have to be induced by nuclear binding which is a small effect at the energies considered. The curve representing the gluino case is seen not to be identical to that of the previous work. In Geant 3 approximately $96 \%$ of the gluino mesons had converted into baryons after travelling through $1 \mathrm{~m}$ of iron 1 ] whereas the corresponding number for Geant 4 is $90 \%$. There is, however a dependence of these curves on the kinematic input distributions. The Geant4 curve was made for $300 \mathrm{GeV} / \mathrm{c}^{2}$ gluino R-hadrons generated with a flat distribution of kinetic energies ranging from 0 to $2 \mathrm{TeV}$. Running the programme at lower energies opens up the phase space for $2 \rightarrow 2$ processes relative to that for $2 \rightarrow 3$ processes. The $2 \rightarrow 2$ processes have a higher fraction of baryon number changing processes than the $2 \rightarrow 3$ processes leading to earlier conversions. 
Looking at the stop and anti-stop hadrons it is seen that the stop and anti-stop hadrons take, on average, a longer distance to change their baryon number due to the lower cross section of the smaller LQS.

\subsection{Energy Loss}

Another quantity influenced by the mass of the LQS of the stop-R-hadrons is the hadronic energy loss as can be seen in figure 7 The LQS in this case carries a smaller fraction of kinetic energy and thus less energy is available in the collision than for the gluino case. This behaviour is clear from the plot. Considering the toy-model in comparison with the parametrised model, one observes that the energy loss grows approximately linearly with the $\gamma$-factor and hence with the energy. This is consistent with the LQS carrying a constant fraction of the total energy given the simplified treatment of the collision in this model. The parametrised model on the other hand shows a somewhat smaller energy loss, the rise of which decreases with energy. As the main difference between the two models is the inclusion of the struck nucleus we can thus here directly see the effect of the nucleus on the energy loss.

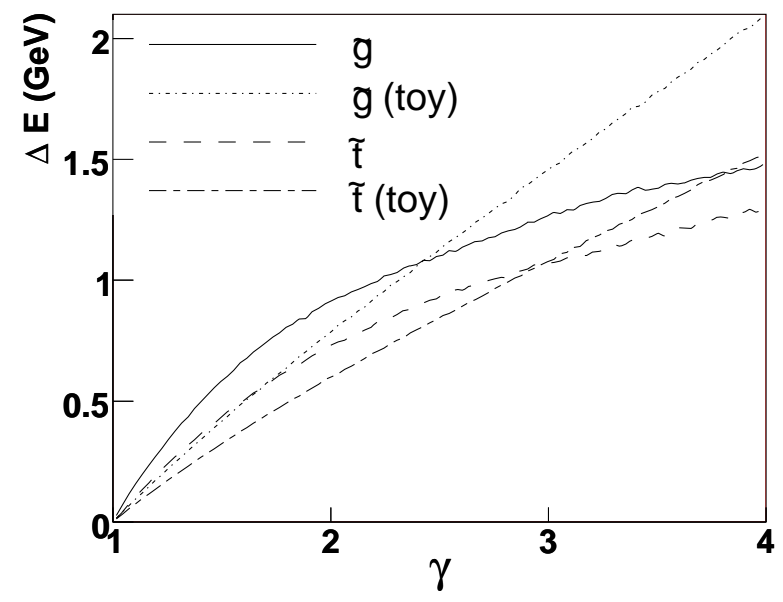

Figure 7: Energy loss per hadronic interaction for gluino hadrons and stop hadrons.

Turning to figure 8 which is a detailed comparison of the energy loss per hadronic interaction between the models in play, one sees immediately that the toy-model is not consistent with the previous work in either a quantative or qualitative sense, as is expected. It does, however, give numbers within the same order of magnitude. We thus see clearly for both distributions the changes in phenomenology induced by the treatment of the struck nucleus and the consequent changes in kinematics. Looking at the parametrised model the energy loss is closer, but not identical, to that predicted with Geant3 model. The difference is expected because the kinematics tratment because the kinematics treatment is somewhat different between the Geant3 and Geant4 versions of this model as discussed 
in section 3.4 .2

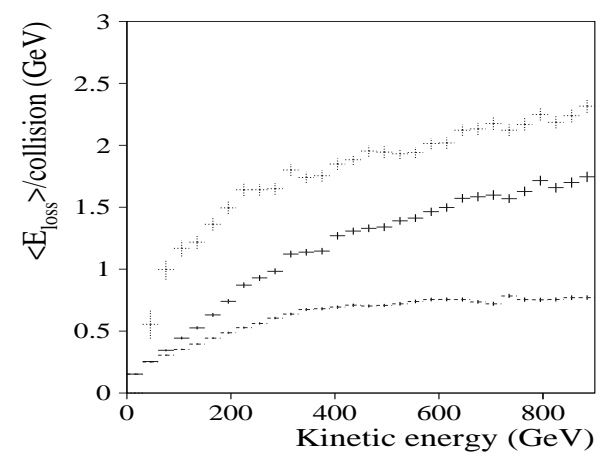

(a) Geant3 1]. The higher set of points is $2 \rightarrow$ 3 processes, while the lower is $2 \rightarrow 2$. The central set of points is the combined one.

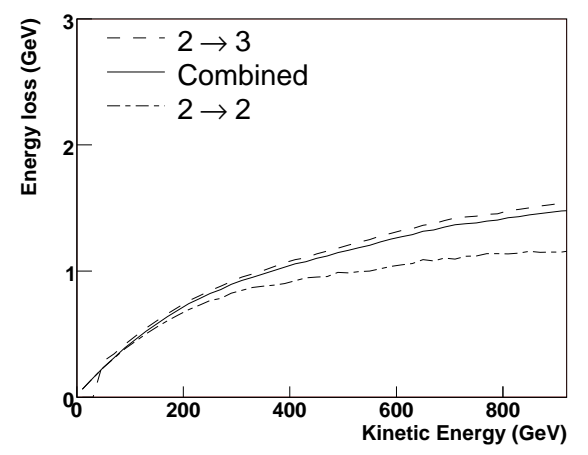

(b) Geant4 - Parametrised model

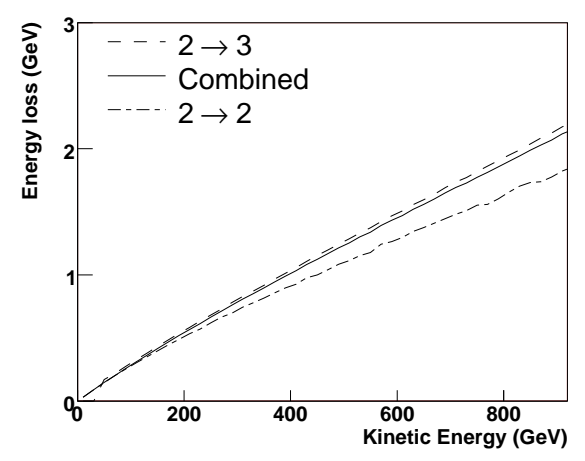

(c) Geant4 - Toy model

Figure 8: Energy loss per hadronic interaction for a $300 \mathrm{GeV} / \mathrm{c}^{2}$ gluino in iron. Both the Geant3 and Geant4 values are shown.

The energy loss is seen to be somewhat higher for the $2 \rightarrow 2$ processes in Geant4 relative to what is predicted in Geant3 while the opposite is true for $2 \rightarrow 3$ processes. The two models are still roughly compatible, however, and nothing here suggests a drastic difference in phenomenology between the two models. To corroborate this observation the energy loss in $1 \mathrm{~m}$ of iron for a $300 \mathrm{GeV} / \mathrm{c}^{2}$ heavy parton is shown in figure 9 . The values were calculated using the parametrised model only.

In the figure one can see both the ionisation and hadronic energy losses, and the combination for a $300 \mathrm{GeV} / \mathrm{c}^{2}$ heavy parton. This is in comparison with the values from Geant3 which are shown in figure 10. The values are seen to be compatible. As the average kinetic energy carried by the LQS is smaller for the stop case than for the gluino case as previously mentioned, the hadronic energy loss is correspondingly smaller and the contribution from ionisation is thus clearly more visible for the stop case than for the gluino case. The effect is amplified by the fact that by simple quark counting a stop sbaryon will more often have double 


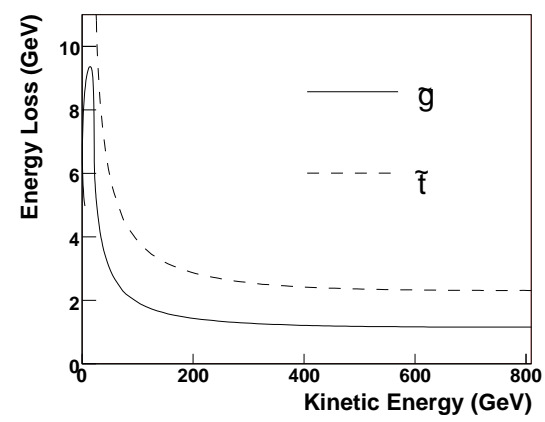

(a) Ionisation loss

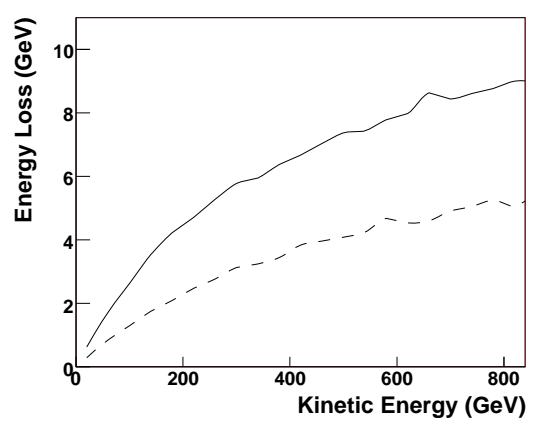

(b) Hadronic energy loss

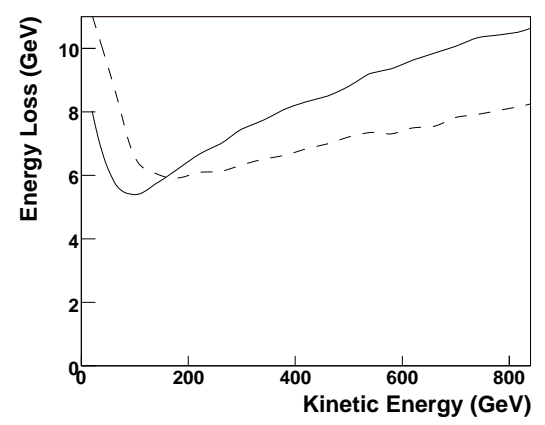

(c) Total energy loss

Figure 9: Absolute energy loss in $1 \mathrm{~m}$ of iron for a $300 \mathrm{GeV} / \mathrm{c}^{2}$ gluino / stop hadron. In the Geant 4 plots the numbers corresponding to gluino hadrons are represented by the fully drawn line whereas the stop hadrons are represented by a dotted line.

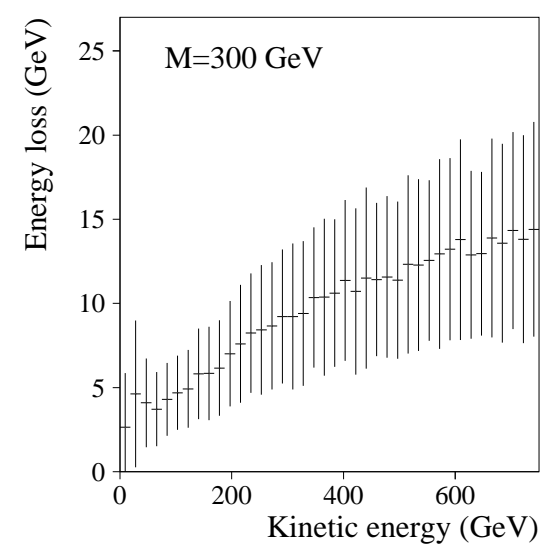

Figure 10: Geant3 hadronic energy loss in $1 \mathrm{~m}$ of iron for a $300 \mathrm{GeV} / \mathrm{c}^{2}$ gluino hadron. 1] 
charge than what is the case for gluino baryons. The charge enters the Bethe Bloch formula squared, leading to a substantially higher energy loss due to ionisation. This is indeed observed in figure 9(a)

We can thus conclude that the physics content of the Geant3 and the Geant 4 models is the same, with one quantitative difference induced by a conceptual difference in the handling of the kinematics.

\section{$4.3 \mathrm{dE} / \mathrm{dx}$}

Turning to a classic observable, energy loss distributions were calculated as a function of distance crossed by the R-hadrons in iron. The simulations were produced in the case of gluino baryons, stop sbaryons and anti-stop mesoninos to simplify matters in relation to section 4.1

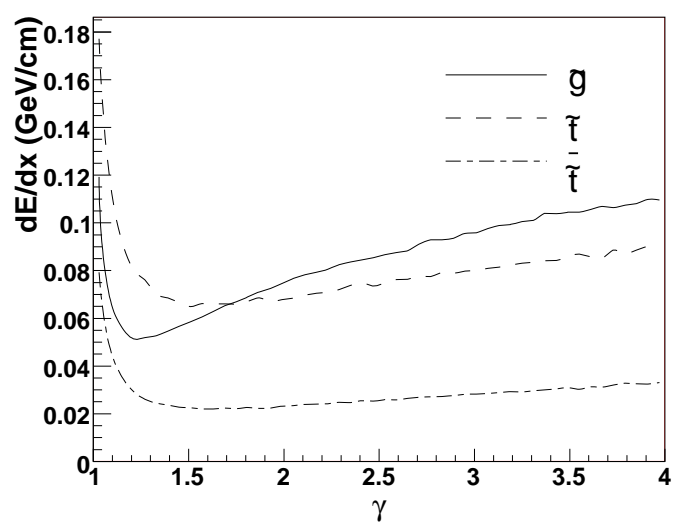

Figure 11: $\mathrm{dE} / \mathrm{dx}$ distributions for a $600 \mathrm{GeV} / \mathrm{c}^{2}$ sparticle mass comparing the stop and anti-stop to the gluino case.

As can be seen from figure 11 there is a difference in the shape of the distribution between the gluino and the stop/anti-stop case while the main difference between the stops and the anti-stops is the overall energy deposition. The difference in shape stems from the cross-over where the hadronic energy loss starts to dominate over the EM energy loss.

Figure 12 shows $\mathrm{dE} / \mathrm{dx}$ for all three kinds of particles where the energy loss has been split into an EM contribution and a hadronic contribution. It is clear from the figure that stops and anti-stops are very similar apart from the overall size of the energy loss. Furthermore the onset of the hadronic energy loss is a lot sharper in the gluino case due to the larger cross section and energy loss per interaction as already discussed in section 4.2

As R-hadrons are often produced in pairs, the difference in normalisation of the $\mathrm{dE} / \mathrm{dx}$ distributions for squarks and anti-squarks could prove to be a powerful estimator to distinguish between a gluino and a squark signal. If dividing a sample of R-hadron candidates into a positively and negatively charged sample resulted in large differences in energy depositions, one would have a powerful argument for a charged colour triplet 


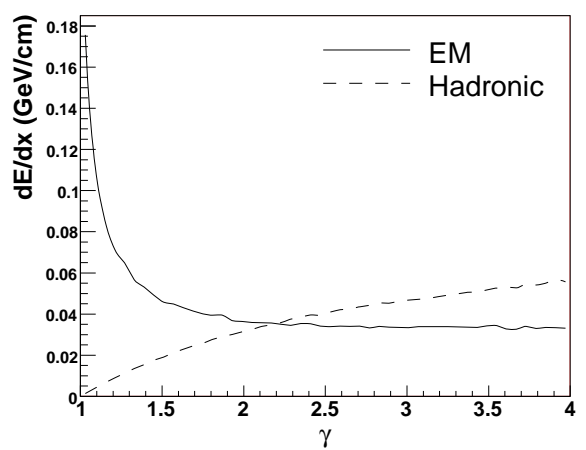

(a) Stops

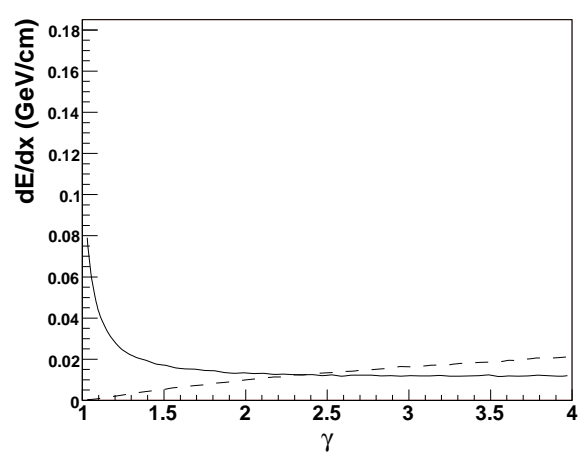

(b) Anti-stops

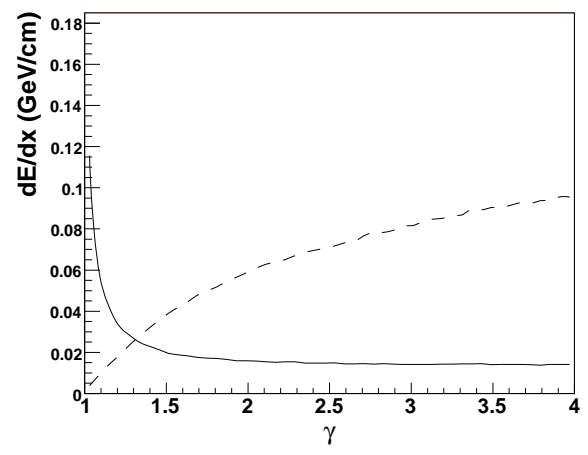

(c) Gluinos

Figure 12: Cross-over between EM and hadronic energy loss for stops and gluinos 
hypothesis. Conversely, if no difference was found or if the samples proved to be ill defined, one would be able to reject the squark R-hadron scenario for at least the case of no oscillations.

\subsection{Shower shapes}

The electromagnetic and hadronic interactions of R-hadrons and their collision reaction products will give rise to showers of secondary and tertiary particles. The profiles of these showers might provide a handle on finding and identifying R-hadrons. Samples were generated in which beams of particles were shot into $10 \mathrm{~m}$ of iron. The particles were generated with a flat distribution in kinetic energy up to $1 \mathrm{TeV}$. Figure 15 shows the energy deposition density transverse to the incident direction of the particles. Hadronic interactions of secondaries were treated using the a standard Geant4 physics list (QGSP).

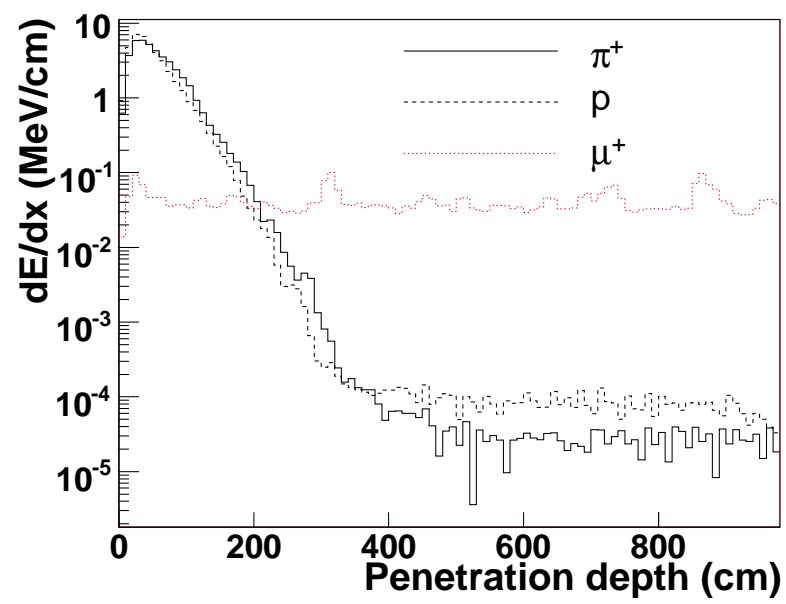

Figure 13: Longitudinal shower profile of pions and protons compared to muons.

Figure 13 sets the scale of the energy depositions. It is seen that hadrons will mostly deposit all their energy within the first $2-3 \mathrm{~m}$ at a scale that is two orders of magnitude beyond typical values for muons.

In figure 14 the various types of $\mathrm{R}$-hadrons are compared to the average muon energy deposition. It is seen that the level of energy deposition is comparable between the muons and the R-hadrons. A detailed comparison between the different species of R-hadrons shows little difference between gluino R-baryons generated with positive or negative charge. This is to be expected as after a few hadronic interactions the R-hadron will have no memory of its initial charge. Also the sign of the charge plays no role neither in the Bethe-Bloch formula or the hadronic interactions. As expected the lower cross section of the anti-stop states lead to a smaller overall energy deposition than for any of the other cases.

Turning to the transverse shower profiles, figure 15 shows the energy deposition density at a distance $r$ from the incident direction of the $\mathrm{R}$ - 


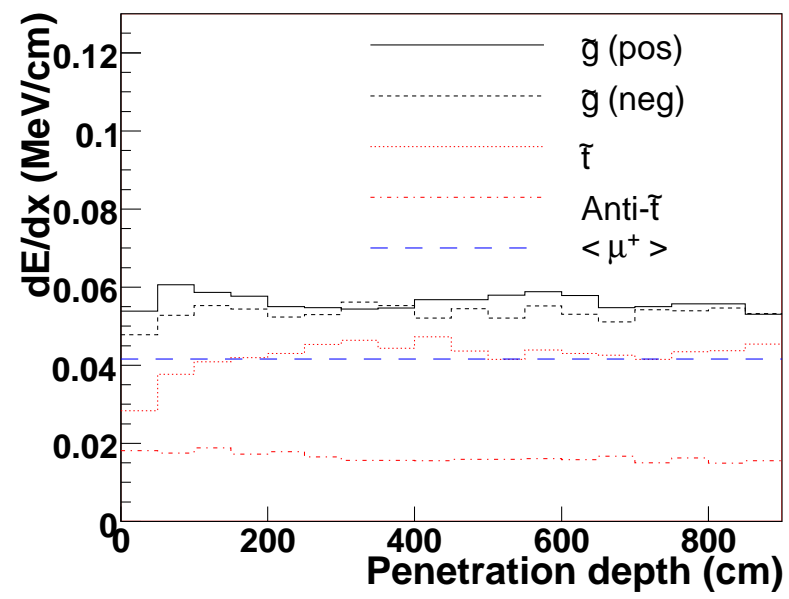

Figure 14: Longitudinal shower profile of muons and $300 \mathrm{GeV} / \mathrm{c}^{2}$ R-hadrons generated with the same flat kinetic energy distribution

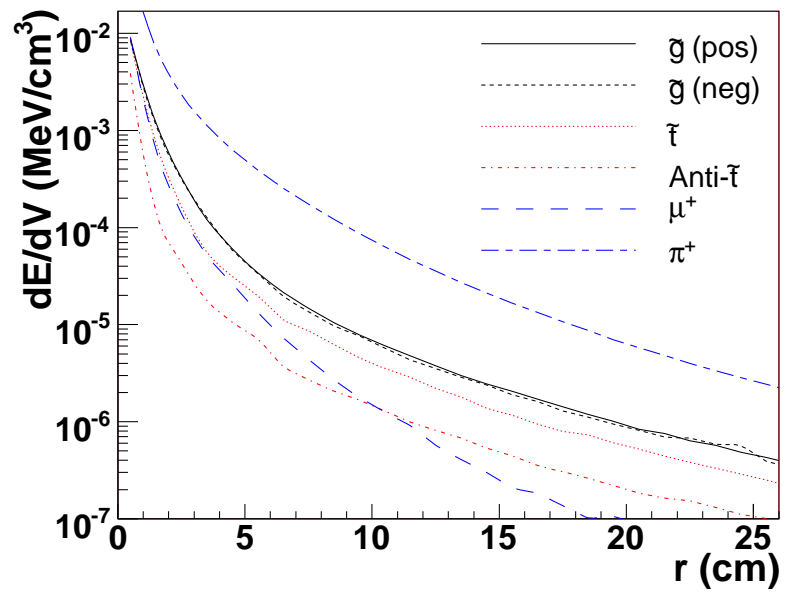

Figure 15: Transverse shower profile for $300 \mathrm{GeV} / \mathrm{c}^{2}$ R-hadrons compared with muons and pions. 
hadron. R-hadrons are compared to muons and pions in order to set a natural scale. From the figure we see again the same differences between the different types of R-hadrons as was the case for the longitudinal profiles. The pions deposit substantially more energy than R-hadrons. This is due to the fact that they deposit all their energy, while the R-hadrons punch through as seen in figures 13 and 14 The muons tend to produce a narrower shower than the R-hadrons. This is consistent with the muons not having hadronic interactions. It thus appears that R-hadron showers deposit substantially less energy than ordinary hadrons and that the energy deposition is very much alike to that of muons apart from the fact that the shower is broader. We may add to this observation the fact that an R-hadron traversing a calorimeter may at times be neutral, leading to gaps in the energy deposition when looking at the longitudinal profile of the shower.

\section{Conclusions}

A method has been presented to simulate the phenomenology of heavy coloured long-lived particles that show up in many novel extensions to the SM. Also with the stop and gluino study we have demonstrated for the first time how predictions can be made to discern between colour triplet and octet states from their interactions with matter. Many observations from this paper may be transferred directly to other models. More novel constructs carrying other combinations of SM charges than quarks and gluons will require careful consideration as to which processes will occur in the interaction with matter. In general, in order to use this model in other scenarios, it is needed to check whether or not the considerations presented here apply.

\section{Acknowledgements}

The authors wish to acknowledge Dennis Wright (SLAC) for his assistance in weeding out the flavour dependence in the parametrised model of Geant4. Also we wish to thank Troels Petersen for supplying the Dalitz plot class used in the toy model of section 3.4.1. Finally we wish to thank Torbjörn Sjöstrand for providing Pythia code to handle gluino and stop hadronisation as well as a list of gluino and stop hadrons with masses and PDG compatible codes.

\section{References}

[1] A. C. Kraan, Eur. Phys. J. C 37 (2004) 91 arXiv:hep-ex/0404001.

[2] M. J. Strassler, arXiv:hep-ph/0607160

[3] M. J. Strassler and K. M. Zurek, arXiv:hep-ph/0604261

[4] S. Agostinelli et al. [GEANT4 Collaboration], "GEANT4: A simulation toolkit," Nucl. Instrum. Meth. A 506 (2003) 250. 
[5] http://cern.ch/r-hadrons/, Usage documentation

[6] M. Fairbairn, A. C. Kraan, D. A. Milstead, T. Sjostrand, P. Skands and T. Sloan, arXiv:hep-ph/0611040.

[7] H. Baer, K. m. Cheung and J. F. Gunion, Phys. Rev. D 59, 075002 (1999) arXiv:hep-ph/9806361.

[8] G. F. Giudice and A. Romanino, "Split supersymmetry," Nucl. Phys. B 6999 (2004) 65 [Erratum-ibid. B 706 (2005) 65] arXiv:hep-ph/0406088.

[9] T. Sjostrand, S. Mrenna and P. Skands, "PYTHIA 6.4 physics and manual," JHEP 0605 (2006) 026 arXiv:hep-ph/0603175.

[10] G. Corcella et al., "HERWIG 6: An event generator for hadron emission reactions with interfering gluons (including supersymmetric processes)," JHEP 0101 (2001) 010 arXiv:hep-ph/0011363.

[11] R. Barbieri, G. Marandella and M. Papucci, "Breaking the electroweak symmetry and supersymmetry by a compact extra dimension," Phys. Rev. D 66 (2002) 095003 arXiv:hep-ph/0205280.

[12] T. Appelquist, H. C. Cheng and B. A. Dobrescu, "Bounds on universal extra dimensions," Phys. Rev. D 64 (2001) 035002 arXiv:hep-ph/0012100.

[13] K. Agashe and G. Servant, "Baryon number in warped GUTs: Model building and (dark matter related) JCAP 0502 (2005) 002 arXiv:hep-ph/0411254.

[14] P. Skands et al., "SUSY Les Houches accord: Interfacing SUSY spectrum calculators, decay packages, and event generators," JHEP 0407 (2004) 036 arXiv:hep-ph/0311123.

[15] H. Fesefeldt, GHEISHA user manual: The Simultion of Hadronic Showers, PITHA 85/02.

[16] A. C. Kraan, "Interactions and Detection of R-hadrons", PhD thesis, University of Copenhagen, 2004

[17] GEANT4 Collaboration, "Physics Reference Manual v. 7.1 Ch. 20", http://geant4.web.cern.ch/geant4/.

[18] D. Wright (SLAC), Private communication, December 2005. 